\title{
Testing Acquisition of GPS / GNSS Location and Velocity to Improve Safety in Autonomous Driving
}

\author{
Karen von Hünerbein ${ }^{1}$, Werner R. Lange ${ }^{1}$ \\ ${ }^{1}$ Lange-Electronic GmbH, Rudolf-Diesel-Str. 29a, 82216 Gernlinden, \\ kvh@lange-electronic.com
}

\begin{abstract}
:
GPS (Global Positioning) and GNSS (Global Navigation Satellite Systems) yield very accurate positioning, velocity and timing, which are crucial for many ADAS (advanced driver assistance systems) and in autonomous driving. 3D locations can be as accurate as a few decimeters, with the help of additional correction methods. In addition a wide range of different sensors, among them optical, LIDAR and RADAR are used to detect obstacles and street marks, GNSS location and trajectory data can be exchanged via wireless data links with other cars (Car2Car) or sent to infrastructure (Car2X), in order to improve early detection of possible accidents, avoid drastic breaking events on the road ahead, traffic jams and detect vehicles around the corner, which are not visible to the driver due to obscuration. This element of telemetry in ADAS and autonomous driving serves to improve safety of all traffic participants and efficiency of driving. In order to guarantee safety of ADAS and autonomous driving, millions of test $\mathrm{km}$ need to be driven on different roads in diverse environments. Especially in city centers and mountaineous areas, GNSS reception can be impaired by obscuration of signals by buildings, bridges, vegetation and mountains and by signals reflected on even surfaces, called multipath. Furthermore, there are many sources of interference, potentially jamming, spoofing and meaconing GPS/GNSS receivers, especially on motorways and in cities. Testing can become very time consuming and costly, especially when driving all the required kms. Thus, it is more efficient to record the GPS/GNSS and interference signal environment during test drives with advanced record and replay systems, to be able to reproduce the realistic signal environment inside the laboratory, enabling repeatable and realistic tests. Such new record and replay systems are able to record GNSS signals on all frequency bands with a high bandwidth and resolution plus many additional sensors synchronously. In this paper we will present the use cases of GPS/ GNSS data in autonomous driving, Car2Car and Car2X, sources of errors and vulnerabilities for GPS/GNSS and appropriate test systems to allow for repeatable and realistic tests in the laboratory. The aim is to decrease time and money spent on testing and to improve the safety of driver assistance systems and autonomous driving.
\end{abstract}

Key words: GPS/GNSS locations, errors and vulnerabilities, safety in autonomous driving, record and replay systems, interference signal environment

\section{Introduction}

In recent years the importance of GPS/GNSS positioning and navigation has grown dramatically in civilian applications, due to the world-wide availability and high accuracy of positions and velocity calculated from GPS/GNSS signals, which can be obtained almost anywhere under the open sky. The positioning, navigation and timing functions (PNT) are widely used, especially in mobile phones and in-car navigation systems, to show the way and the current location. In the last few years, more and more research and development effort have been invested into Car2Car (V2V), Vehicle to Infrastructure (V2X),
Driver assistance functionality (ADAS) applications, and more recently into fully autonomous driving. PNT provided by Global Navigation Satellite Systems (GNSS) is key for most of these applications and technologies $[1,2,4,5]$.

Car to Car communication (C2C or V2V), is a technology under development, employing the IEEE $802.11 \mathrm{p}$ standard at $5.9 \mathrm{GHz}$ in Europe, for the exchange of messages in between cars, about location, heading, trajectory, special events of the current vehicle, which are being broadcast to all vehicles in the vicinity. The goal of this technology is to increase awareness and safety for each vehicle on the road by allowing to foresee dangerous situations and issue alerts 
to the driver in case of incidences, e.g. on a motorway, a car fully breaking on the road ahead that may be concealed by bigger transport lorries. Other goals of $\mathrm{V} 2 \mathrm{~V}$ are to enhance traffic flow, eco-driving, efficiency and gaming/entertainment. One important component of the messages is the location calculated from GPS/GNSS signals. Accuracy needs to be very high in the 1-2 $\mathrm{m}$ range in order to be able to distinguish on which lane a car is moving, breaking or static. [2,3]

A similar technology concept is vehicle to Infrastructure communication (V2I) and vice versa, e.g. informing drivers ahead of time about obstacles, red traffic lights and other vehicles or pedestrians approaching sideways on a crossing, possibly invisible by buildings and vegetation. Again, accuracy of location needs to be high for the prediction of dangerous situation, and for distinguishing between critical and non-critical events [2,3]. Both types of communication represent special, challenging cases of telemetry by using remotely acquired and transferred data to improve driver awareness, traffic flow and traffic safety.

ADAS, Advanced Driver Assistance Systems, are electronic applications in cars to assist the driver in all sorts of driving situations, some of them potentially dangerous, where additional information can be helpful, e.g. blind spot monitoring, lane departure warning, Collision Avoidance System, Parking Assistant, wrong way driving warning [4]. These systems can operate stand-alone in the car and are based on an array of different sensors, such as cameras, LiDAR (light detection and ranging) [7], automotive RADARs, ultrasound, odometers, accelerometer, gyroscopes, and GNSS. Cameras provide an all round view to the driver. LiDAR, ultrasound sensors and RADARs serve to measure distances to obstacles and objects around the vehicle. Wheel speed sensors, called odometers, deliver information about speed, and dangerous states, such as blocked wheels, or wheels, which lost their grip. Accelerometers provide accelerations, speed changes in different directions, and gyroscopes indicate heading and heading changes. The latter two are also called inertial sensors used for inertial navigation $[5,6,12]$. In order to integrate all these different type of sensor information, intelligent sensor fusion algorithms have been developed with the challenge of correctly interpreting the measurement results of various sensors and to deal with conflicting information [8].

In autonomous driving a great variety of different sensors is employed, for gaining maximum information. GNSS positioning is a crucial source of location information with extremely demanding requirements, such as non-stop availability (24h/7d a week), very high accuracy of location, and fail-safe-operation. All of these conditions need to be fulfilled even in challenging environments to ensure the safety of passengers, other vehicles, pedestrians, cyclists and infrastructure. Many environments pose particular challenges to GPS/GNSS signal reception, as these signals are transmitted by moving satellites, which are distributed across the visible sky. Some satellites are located at a low elevation, so that their GPS/GNSS Signals can be attenuated or blocked by vegetation, buildings and mountains. In certain environments a lot of multipath is present, caused by reflections of GPS/GNSS signals by even surfaces such as glass, metallic and water surfaces. In this case, there are several signals for the same satellite arriving from various directions, they are weaker in signal power than the LOS signals, travel longer non-direct paths, and they contain exactly the same information in the navigation message as the other signals [9]. In this case, the receiver algorithm has to decide, which of the multiple signals from each satellite it should use for the PNT fix.

GPS/GNSS signals are vulnerable, because they arrive at the surface of the Earth with a very low signal power of -120 to $-130 \mathrm{dBm}$, so low that it is usually buried inside the thermal noise. Thus GNSS signals are easy to interfere with and vulnerable to different types of effects, including atmospheric disturbances, multipath and malicious spoofing. Interfering radio signals in the L-band can be emitted and generated unintentionally, e.g. by defect devices or intermodulation effects of several RF transmitters, or different transmitter antennas installed on top of roofs of large sea vessels for a variety purposes, e.g. for mobile satellite communication and RADAR. They can also be emitted with the intention of jamming or spoofing, which is providing false signals to the receiver, providing misleading information [9, 20,21]. All these disruptive effects and degradations need to be considered, mitigated and verified at all development stages by thorough testing.

To increase accuracy, availability and integrity, GNSS correction data are used and additional sensors are integrated into the onboard navigation system. Much higher accuracy can be achieved by a method called Real Time Kinematic (RTK), using carrier phase measurements, where a mobile reference station transmits GPS/GNSS correction data to a rover receiver over the air. It is based on "carrierbased ranging and provides ranges and 
positions that are orders of magnitude more precise than those available through codebased positioning." [19] The horizontal position accuracy can be improved to about $2-3 \mathrm{~cm}$ in real-time. Correction data are also provided by national CORS Networks (Continuously Operating Reference Station), with stationary reference stations, which continuously monitor all GNSS satellites and gather integrity information and pseudorange corrections for their specific location. These data can lead to a significant improvement of the accuracy and integrity of the position fixes up to $200 \mathrm{~km}$ from the reference station. "Rovers determine their position using algorithms that incorporate ambiguity resolution and differential correction. Like DGNSS (Differential GNSS), the position accuracy achievable by the rover depends on, among other things, its distance from the base station (referred to as the "baseline") and the accuracy of the differential corrections. Corrections are as accurate as the known location of the base station and the quality of the base station's satellite observations. Site selection is important for minimizing environmental effects such as interference and multipath, as is the quality of the base station and rover receivers and antennas." [19]. RTK is a method for real-time correction. There are also methods for post-processing data to improve accuracy, if it is not needed in the field, such as postprocessed kinematic, allowing for more convenient calculation in the lab. However, in autonomous driving highly accurate positioning is required in real-time during the drive so that post-processing is not an option.

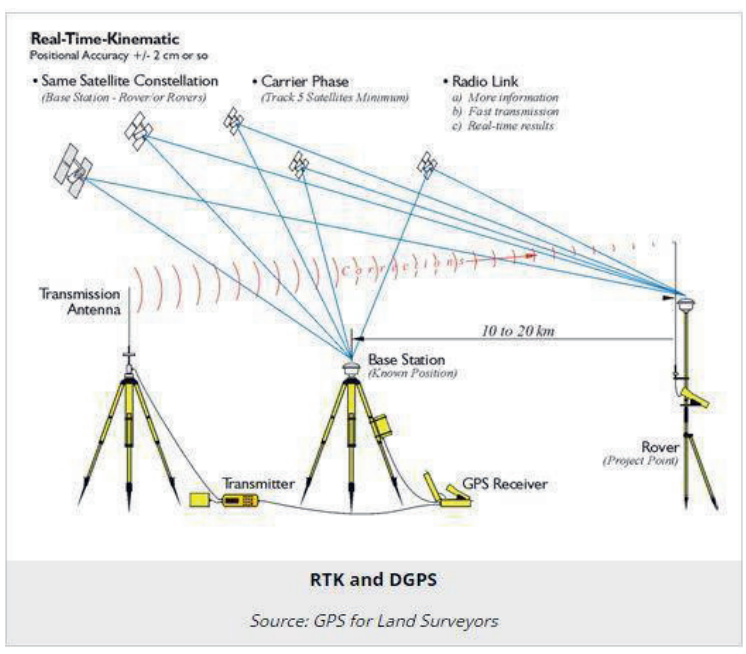

Fig. 1. Overview Real Time Kinematic [13]

Another type of correction data are broadcasts from special geostationary satellites. These systems are called Satellite Based Augmentation Systems (SBAS). Some provide data in the public domain free of charge [e.g. EGNOS the European Geostationary Overlay Service, 16], others are commercial systems, e.g. Starfire or Omnistar $[17,18]$. Correction data of the publicly available SBAS can improve accuracy to 1-3 $\mathrm{m}$, which is sufficient for some ADAS, but not for autonomous driving. The commercial SBAS systems are able to provide accuracy in the sub-dm range, down to $5 \mathrm{~cm}$ [22].

Higher availability, accuracy and integrity of positioning can be achieved by a combination of GPS/GNSS with inertial measurements units (IMU), containing different inertial navigation sensors (INS). "Inertial navigation is a selfcontained navigation technique in which measurements provided by accelerometers and gyroscopes are used to track the position and orientation of an object relative to a known starting point, orientation and velocity. Inertial measurement units (IMUs) typically contain three orthogonal ... gyroscopes and three orthogonal accelerometers, measuring angular velocity and linear acceleration respectively. By processing signals from these devices it is possible to track the position and orientation of a device." [citation from 10]. "GPS/Inertial systems combine the interference mitigation, high bandwidth and low noise benefits of inertial measurements with the long-term stability of GPS into a self-calibrating, higher accuracy blended navigation solution" [11]. Inertial systems alone are not able to maintain a stable accurate position fix over a long-time, as they drift too much. They are well able to bridge short gaps caused by GPS/GNSS outages lasting several seconds to a few minutes. Thus INS provides greater continuity and full availability, while GPS/GNSS provides stability of PNT accuracy. Both systems complement each other with their respective strengths $[5,6$, 11 , 12]. According to automotive experts, GNSS/INS Positioning, Navigation and Timing will be a major building block for highly automated driving [1].

\section{Importance of Testing}

Because of the GPS/GNSS vulnerabilities, and in order to check the performance of the integrated sensor system and sensor fusion algorithms, thorough testing of all components and of the complete autonomous vehicle with all integrated sensors and algorithms is required. Test of components and sub-systems can be carried out in the laboratory. To verify safety, the fully integrated autonomous verhicle must successfully complete millions of $\mathrm{km}$ driven on real roads around all sorts of different environments: urban, sub-urban, mountaineous area, motor-ways, small streets, and many more. 
In order to maintain correct navigation and timing, it is crucial that the receiver algorithm has functions to detect and cope with dangerous and unexpected events. These functions need to be tested, too, to guarantee continued operation, even in case of external error sources. When improving software algorithms or installing other countermeasures, systems need to be retested to verify and quantify and the improvement.

The easiest way of testing is a live sky test with live GNSS signals outside buildings. Advantages are immediate availability and realistic signal environment. Disadvantages are high time consumption, high cost and lack of repeatability, because all GNSS signals vary a lot with time, as the GNSS satellites are moving fast in their orbits. They also vary with weather, 3D terrain and different disruptive factors, as described above. Thus the tests under live-sky conditions can only convey part of the picture and do not allow for controlled and repeatable testing.

More systematic testing can be performed in the laboratory. A lot more tests can be run in the laboratory than in the field, improving the results and giving a chance to fully asses the strengths and weaknesses of the GNSS based positioning and navigation [26].

Testing in the lab can save a lot of time and will improve the overall reliability and functionality of the GNSS based navigation unit and answer questions like: How quickly does the navigation unit recover after coming out of a tunnel? How well does it hold on to a signal, e.g. near buildings and under significant tree cover? How robust is the PNT solution when stressed by real-world threats? How accurate is the PNT solution in different types of environments? [26].

\section{Record and Replay of GNSS signals}

Record and Replay of GNSS signals has been established as a valuable test method, with signals being received and captured in a static or dynamic measurement like a test drive. The signals are then converted to IF and stored on a hard disk, and later replayed in the lab after upconversion from the IF back to the RF signals, faithfully reproducing any propagation effects. [9, 15].

The advantages are that the recordings can fully capture real signal environments with complex errors including obscuration and multiple reflections, called multipath, real-world fades and in-band interference. Signals from multiple test drives at difficult locations, with a known GPS/GNSS reception problem, can be captured and used for tests, with no need to revisit the real location. The replay tests are fully repeatable, as the same signal environment is reproduced during each replay, at the same time and date $[9,14,15]$.

On the other hand there is virtually no control over the signals and the error conditions in the recording, except that some attenuation can be applied. Contrary to GNSS simulators, time and date of the test drive cannot be changed, nor the amount of satellite signals present in the recording, nor the signal parameters and the navigation message.

For every new location or date a new test recording is required. The recorded error conditions captured in the recording are usually unknown, unless the user has additional information about special conditions from external sources, like space weather reports. Test drives and recordings are easy and cost effective for stationary and land vehicle based receiver and allow Software and Hardware testing including system trials, algorithm studies and iterative algorithm development, interference and jamming recording and monitoring.

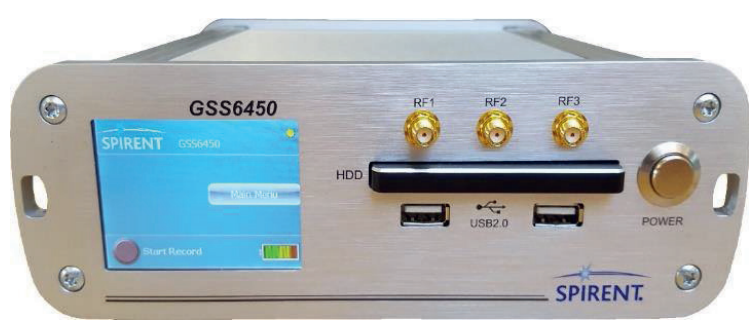

Fig. 2. Front view of Record and Replay System

One example of a record and replay system is the Spirent GSS6450 [14,24,25]. The record / replay system (RPS) GSS6450 is a portable unit with a weight of $2.2 \mathrm{~kg}$ capable of recording 4 GNSS bands simultaneously at all L-band frequencies, including IRNSS (Indian Regional Navigation Satellite System) L5, SBAS L1 and L5, Inmarsat based correction services, QZSS (Quasi Zenith Satellite System) L1, L5, L6, Beidou B1, B2, B3, GLONASS L1,L2,L3 and Galileo E6. In short it will record all major GNSS bands via $1 \mathrm{RF}$ input port and many SBAS and regional service signals. The latest version of the GSS6450 is capable of recording signals in the GNSS, WIFI and LTE band, simultaneously, via 3 RF input ports, the second RF port records $690-2400 \mathrm{MHz}$ and the third one records $100 \mathrm{MHz}-6 \mathrm{GHz}$ for WiFi bands at 2.4 $\mathrm{GHz}$ and $5.0 \mathrm{GHz}$ [24]. During recording, the RF signals, are downconverted, digitized and stored at IF. During playback, the IF signal is recreated and then up-converted to RF at the relevant GNSS frequency using the same builtin oven controlled local oscillator (OCXO) as used to record the data for minimum phase 
noise." $[9,14]$. RF signals can be recorded at 4 , 8 or 16 bit for quantization and at 10, 30, 50, 60 $\mathrm{MHz}$ and $80 \mathrm{MHz}$ bandwidth [24]. There are throughput limitations at 8 and 16 bits together with the 50, 60 and $80 \mathrm{MHz}$ bandwidth, limiting the amount of channels that can be recorded simultaneously to 1-2 [14]. The record and replay system contains an OCXO for record and playback for high frequency stability. It is very small with a size of $21^{\star} 20 \mathrm{~cm}$, with a large storage capacity of 2-4 TB internal and external SSD [14]. USB 3.0 is supported allowing data transfer to or from external drives [24].

The major advantage of this record and replay system is the 16 bit depth for quantization of I and $Q$ each, allowing to capture GNSS signals even at high jamming powers. Most other portable record and replay systems have a 2 bit quantization, which is suitable for general GNSS signals with a $12 \mathrm{~dB}$ dynamic range. "However it will not be good under jamming / interference scenarios as the interference 'soaks' up the dynamic range reducing GNSS resolution as described" and it is also "limited in the ability to capture Multipath and Fading effects for Real environments where small signal perturbations cannot be captured by the 4 levels of 2 bit sampling... 16 bit sampling overcomes this by increasing the 4 levels to 65536 levels." [personal communication by Julian Kemp]. Greater bit depth allows better resolution of GNSS signals and greatly increases this dynamic range both for jamming and for multipath and fading effects, to $21 \mathrm{~dB}$ at 4 bit I and 4 bit $Q$, to $45 \mathrm{~dB}$ at 8 bit I and 8 bit $Q$ and to $80 \mathrm{~dB}$ at 16 bit I and 16 bit $Q$.

Sampling rates are $10.23,30.69$ or $51.15 \mathrm{MHz}$, synchronous recording rate for external data is $300 \mathrm{kbps}$ at $10.23 \mathrm{MHz}, 900 \mathrm{kbps}$ at $30.69 \mathrm{MHz}$ and $1500 \mathrm{kbps}$ at $51.15 \mathrm{MHz}$, asynchronous recording rate for external data is 4800-115200 baud. [14] The reference oscillator is an OCXO with a frequency of $10.23 \mathrm{MHz}$, to allow direct generation of the wanted GNSS frequencies. There are two RF outputs: one normal RF output with a standard GNSS RF signal strength (nominal $-130 \mathrm{dBm}$ for GPS L1), and one high power output at the back of the test system with around $-80 \mathrm{dBm}$ nominal [14]. In addition there is a $10 \mathrm{MHz}$ Reference IN port, allowing to input a source of precise timing. The better the timing, the better the accuracy of the GNSS Position, Navigation and timing solution.

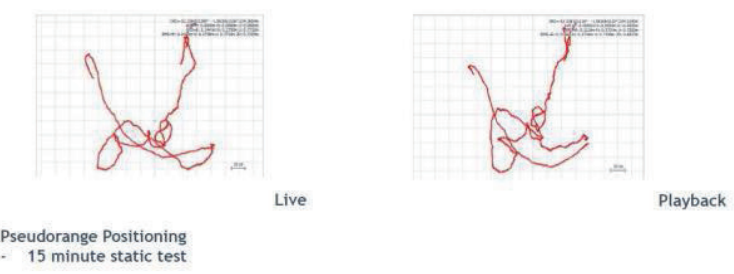

Fig. 3. Comparison of pseudorange position fixes with live sky signals and replayed GNSS signals measured with a Novatel GNSS receiver at $10 \mathrm{~Hz}$ update rate. Each grid cell's width and height corresponds to $10 \mathrm{~cm}$ [25]

The system contains two types of attenuators for replay: single channel attenuators with a total attenuation of $9 \mathrm{~dB}$ in $0.5 \mathrm{~dB}$ steps and a general attenuator for all channels with a total attenuation of $31 \mathrm{~dB}$ in $1 \mathrm{~dB}$ steps $[9,14]$.

"The GSS6450 is fully integrated and no PC or external drives are required" [14]. It can be controlled from the front panel, over WiFi, from the webserver or via scripts. "Scripts allow inclusion of automatic test routines" [14]. Major actions and settings are available from the front panel with a LCD touch screen. Remote control is possible via a VNC (Virtual Network Computing) server and HTTP (Hypertext Transfer Protocol) messages. VNC is a software displaying the screen display of a remote computer on a local computer. This record/replay system can be controlled by devices like ipad, iphone, tablet and Laptop via a WiFi interface." [14]. Additional control options are inclusion of event markers in the recorded file and the option to start replay at any point in the file.

Two units can be joined together to run in a master/slave configuration and thus can record/ playback 8 GNSS frequencies synchronously.

External data can be recorded synchronously and asynchronously. The wide range of external data sources includes: audio, CAN (Controller Area Network) bus data, timing pulses, NMEA (National Marine Electronics Association) data, IMU (Inertial Measurement Units) and other sensors. Up to 8 synchronous inputs can be stored, that is two per recorded GNSS frequency $[9,14]$. Synchronous recording of external data, such as CAN bus data and IMU data, is possible as serial datastreams. Such data are stored inside the GNSS file with GNSS raw data, and can be replayed synchronously, but not retrieved separately. In addition, recording of 4 different video streams parallel to GNSS signals is possible via webcams, and the video signals can be replayed within $0.5 \mathrm{sec}$ of the GNSS signals, 
with a potential drift rate of 0.25 seconds per hour. [14]

External data can be also stored asynchronously, via the serial port, resulting in additional files, stored separately from the GNSS signal data, which can be easily edited and modified, e.g. NMEA data or WiFi data. Timestamps can be added to the serial data [14]. In the new model there is an integrated High Speed CAN bus interface for 2 highspeed CAN interface devices, eliminating the need for an external box. It provides physical recording of the signals without acknowledgements and bus arbitration. [24].

In addition, the RPS GSS6450 contains an L1 only GPS/GNSS receiver, integrated together with an internal LINUX controller, allowing to cross check visible satellites and carrier to noise levels. The internal GSS receiver uses GPS and Galileo L1 signals with either Beidou L1 signals or GLONASS L1 signals and is able to record NMEA and display C/No levels on the front panel display [14].

The GSS6450 can be used for testing of highly accurate PNT: a) by recording any corrections b) by using 2 RPS units, one as a rover and one as a base station. The RPS unit serving as basestation can replay the GNSS signals to a high quality geodetic GNSS receiver, which calculates the corrections and transmits the correction data to the rover. For this procedure carrier phase positioning is used, which relies on high quality carrier phase measurements. In two test campaigns it was shown that RPS units provide high quality carrier phase observations, and can be used for RTK and post processing carrier phase purposes [25, 27].

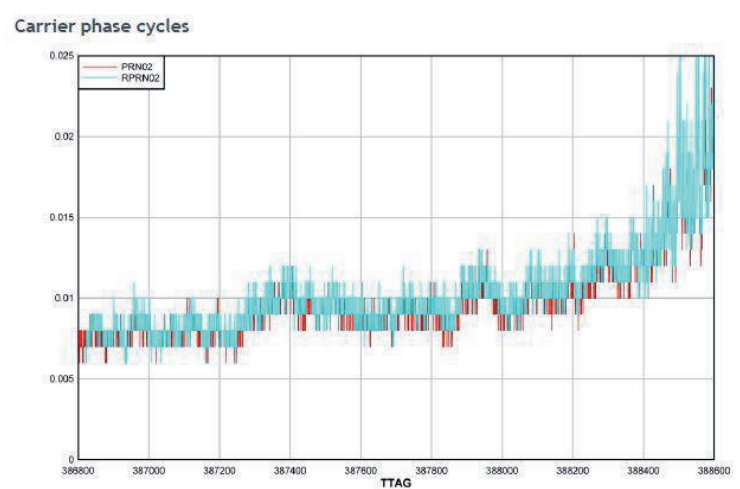

Fig. 4. Comparison of phase noise measurements of live signals (red) and recorded and replayed signals (blue) Plot for GPS SV02 measured with a Novatel receiver, parameter ADRStd on $y$-axis from $0-0.025$ [25]

\section{Advantages for autonomous driving:}

As described above, a multitude of sensors is employed in autonomous driving, in order to ensure comprehensive awareness of the environment. This record and replay test system, allows to record signals of many different key sensors in parallel, synchronously with the GPS/GNSS signals, and replay them repeatedly in the laboratory.

The advantages are that the recordings can fully capture real signal environments with complex errors including obscuration and multiple reflections, called multipath, real-world fades and in-band interference. Signals from multiple test drives at difficult locations, with a known GPS/GNSS reception problem, can be captured and used for tests, with no need to revisit the real location. The replay tests are fully repeatable, as the same signal environment is reproduced during each replay, at the same time and date.

\section{Benefits}

- Recording of all GNSS bands with a high bandwidth in combination with inertial sensors

- All in-band signal effects, external factors, and interference signals are captured like multipath, obscuration, atmospheric delays, interference signals

- Synchronous recording of external sensors and units, e.g. IMUs, CAN bus data [14]

- Highly synchronous replay of synchronously recorded data

- Additional data can be sampled via serial port and stored internally

- Video and audio data

- Connection of two RPS units for recording even more frequencies and sensors in parallel

- Internal GPS receiver records NMEA data for comparison, with laboratory results

- Full repeatability

- High dynamic range, due to high bit depth of digitization

- High quality of signals, low phase noise

- Portability, relatively low power consumption, so that it can be run on a car battery without exhausting it, as observed in test drives by TU Braunschweig.

\section{Drawbacks}

- No control over GNSS signals: signals cannot be modified, only attenuated

- Error effects are uncontrolled and unknown except that it is possible to set event markers (e.g. passage through a tunnel or deep valley) and to cross check the data with a video 
stream, so that external structures can be seen.

- Throughput limitations at high digitization bit depth, when combined with high bandwidth

- Large recorded data volume

- Small losses in signal power due to digitization

Recording the GNSS signals together with the external sensors takes a lot of storage space, so that recording time is fairly limited when using the internal SSD only and when recording 3 or 4 frequencies of GNSS signals simultaneously with a bandwidth of 30 or $50 \mathrm{MHz}$ each. This limitation can be mitigated by the option to use 2 SSDs with 2 TB each and by the possibility to attach a 8 TB external memory. However, attaching external memory makes the setup more bulky and less easy to handle during a test drive [23].

There is a certain loss in GNSS signal strength due to digitization. When 2 bits are chosen for digitization 2 bits for I and $Q$ component of each frequency, this results in a $1.5 \mathrm{~dB}$ loss of signal strength overall. This loss is much smaller, when recording with 8 or 16 bit digitization. In addition loss can be compensated for by replaying the GNSS signals with $+10 \mathrm{~dB}$ or +20 $\mathrm{dB}$ amplification. Alternatively, it is possible to switch on an AGC compensation to replay the signal within $3 \mathrm{~dB}$ of the original signal level. [24].

\section{Examples of Test Use Cases:}

- Interference and Jamming

- GPS/INS sensor fusion

- Algorithm proving

- Multipath

\section{References}

[1] M. Baus, Safe GNSS/Inertial Positioning for Highly Automated Driving. Presentation. Munich Satellite Navigation Summit (2018)

[2] Car2Car Consortium, The Car-2-Car Communication Consortium Roadmaps beyond Day-1. Presentation. (2016) Retrieved from https://www.car-2-car.org/index.php?id=214

[3] VW press release: https://www.volkswagenmedia-services.com/en/detailpage/-/detail/Withthe-aim-of-increasing-safety-in-road-trafficVolkswagen-will-enable-vehicles-tocommunicate-with-each-other-as-from2019/view/5234247/7a5bbec13158edd433c6630f 5ac445da?p_p_auth $=198 \mathrm{AdbHg}, 28^{\text {th }}$ June, 2017
- Sensitivity

- Genuine Equatorial Scintillation

-Testing for BeiDou-2 B1 and GPS, GLONASS, Galileo [25]

\section{Conclusions}

In this paper we have described the crucial role of GNSS positioning and navigation for Car2Car, Advanced Driver Assistance Systems and autonomous driving. Multisensor systems and GNSS correction data are necessary to achieve the stringent requirements for safety, accuracy and reliability. In a navigation unit for autonomous driving, a major building block will be a combination of GNSS and inertial sensors (INS). GNSS signals are vulnerable and there are many different error sources, degrading or even preventing GNSS signal reception. Thus, thorough testing is required. Drive tests are necessary, but time-consuming and not repeatable. A GNSS Record and Replay System is capable of recording and replaying signals from all GNSS bands with a high signal quality and low phase noise, including all in-band error effects on the GNSS signals such as interference, multipath and atmospheric effects. Additional signals, e.g. inertial sensor data, can be recorded and replayed synchronously. This allows repeatable testing in the laboratory with different sensors and can save a lot of test drives speeding up development and verification.

\section{Acknowledgements}

We would like to thank Dave Belton, INS, for permission to use figures 3 and 4, Jan van Sickle for permission to use figure 1, Ali Soliman and Romain Zimmermann, Spirent, for valuable advice.

[4] L. Junge, FAMOS Galileo for Future Automotive Systems. Conference AAET2012, 13th Braunschweiger Symposium, 8th-9th Feb 2012 Retrieved from http://www.dlr.de/rd/Portaldata/28/Resources/ dokumente/rn/satnav/4.GalKonf05_Junge.pdf

[5] M. Gäb, Ein Beitrag zur Stützung eines Software GNSS Empfängers mit MEMS-Inertialsensoren. PhD Thesis, May 2016, Institut of Navigation of the University Stuttgart (2016)

[6] G. Falco, M. Pini, G. Marucco, Loose and Tight GNSS/INS Integrations: Comparison of Performance Assessed in Real Urban Scenarios. Sensors (2017), 17, 255; doi:10.3390/s17020255

[7] Y. Gao, S. Liu, M. Atia, A. Noureldin, INS/GPS/LiDAR Integrated Navigation System for Urban and Indoor Environments Using Hybrid Scan Matching Algorithm. Sensors (2015), 15, 23286-23306; doi: 10.3390/s150923286. 
[8] B. Reuper, M. Becker, S. Leinen, Performance Assessment of Automotive Localization Algrorithms via Metrics on a Modular Level. International Symposium on Certification of GNSS Systems \& Services, 05-06 July 2017, Darmstadt, Germany (2017)

[9] K. v. Huenerbein, W. Lange, Monitoring of the interference environment on large vehicles with a network of highly accurate record and replay systems synchronised by a very precise timing unit, European Navigation Conference, Lausanne, Switzerland, 9th -12th May, 2017

[10] Retrieved from https://en.wikipedia.org/wiki/ Inertial_navigation_system

[11] N. Fedora, C. Ford, P. Boulton, A Versatile Solution for Testing GPS/Inertial Navigation Systems, Proceedings of the 21st International Technical Meeting of the Satellite Division of The Institute of Navigation, September 16 - 19, 2008, Savannah International Convention Center: 1227 - 1236 (2008)

[12] A.D. King. "Inertial Navigation - Forty Years of Evolution" (PDF). GEC Review. General Electric Company plc. 13 (3): 140-149 (1998). Retrieved from: http://www.imar-navigation.de/ downloads/ papers/inertial_navigation_introduction.pdf

[13] Jan Van Sickle, GPS for Land Surveyors. Book. Taylor and Francis Group. (2008) Figure 1 retrieved from: https://www.eeducation.psu.edu/geog862/node/1828

[14] Spirent Communications GSS6450 MultiFrequency Record\&Playback System. MS3098 Datasheet with Product Specification. Issue 2-03, (2018)

[15] S. Hickling, T. Hadrell T, "Recording and Replay of GNSS RF Signals for Multiple Constellations and Frequency Band" Proc. of ION Conference, Nashville,TN; (2013)

[16] U. Celestino et al. "Expanding EGNOS Horizons in North Africa and the Middle East", Inside GNSS March/April 2015, vol. 10(2), pp. 44-52; (2015)

[17] T. Sharpe, R Hatch, F. Nelson. "John Deere's Starfire System: WADGPS for Precision Argriculture", (2006) Retrieved from: https://web.archive.org/web/20060711194114/ http://www.navcomtech.com/docs/ StarFireSystem.pdf

[18] http://www.omnistar.com/

[19] Novatel: https://www.novatel.com/an-introductionto-gnss/chapter-5-resolving-errors/real-timekinematic-rtk/

[20] K. Hünerbein, W. Lange. Real Life Evidence for Spoofing and Jamming of GNSS Receivers, Conf. Proc. of CerGal, DGON Conference, Darmstadt, Germany, $7^{\text {th }}-8^{\text {th }}$ July, 2015.
[21] M. Stanisak, K. Hünerbein K, U. Bestmann, W. Lange, "Measured GNSS Jamming Events at German Motorways", Proc. of POSNAV ITS, DGON Conference, Berlin, Germany, $5^{\text {th }}-6^{\text {th }}$ July, 2016.

[22] Spirent Communications, Testing MultiFrequency, Survey-Grade GNSS Receivers. Case Study (2014)

[23] K. Huenerbein, W. Lange (2015) Multi-Sensor vehicle testing: Recording Inertial Sensors via CAN bus in Combination with Recorded GNSS RF signals. DGON Conference Proceedings: Inertial Sensors and Systems, $22^{\text {th }}-23^{\text {th }}$ Sept 2015, Karlsruhe.

[24] Spirent Communications, GSS6450 - 3RF- V3 SW Improvement - Automotive Focus. Presentation, Issue 1.00 (2018).

[25] Spirent Communications, GSS6450 Performance Analysis Summary. Presentation, (2018).

[26] Spirent Communications. How to construct a GPS/GNSS Test Plan. A guide for engineers integrating GPS/GNSS capabilities into new devices. Whitepaper (2016).

[27] C. Fuente, "GSS6425 Multi-Frequency Recordlplayback - 50MHz RTK Results", Presentation (2016). 